\title{
Analysis of Possible Concept Solutions of Chain Drives
}

Milan Rackov ${ }^{1, *}$, Siniša Kuzmanović ${ }^{1}$, Ivan Knežević ${ }^{1}$, Maja Čavić1, Marko Penčić ${ }^{1}$, Dijana Čavić ${ }^{1}$, and Nicolae Florin Cofaru ${ }^{2}$

1 University of Novi Sad, Faculty of Technical Sciences, Trg Dositeja Obradovića 6, 21000 Novi Sad, Serbia

2 "Lucian Blaga" University of Sibiu, Faculty of Engineering, Str.Emil Cioran Nr.4, 550025 Sibiu, Romania

\begin{abstract}
Possible concept solutions of chain drives are analysed in this paper in order to point out the benefits of chain drive application and, in particular, the possibility of application of multilevel chain drive. The paper also analyses the possibility of replacing the gear drives with chain drives, which, in the case of short-term drive and using the same direction of rotation, enables a slightly simpler drive solution. The paper doesn't consider difference between the roller and gear chains application because these two types of chains can be used very successfully. Also, the possibility of using timing belt drive is not considered, but it can be also used very successfully in this type of drive, but only as a transmission of motion.
\end{abstract}

\section{Introduction}

It is well known that chain drive systems are effective power transmission choice and they can be used similar as gear drives and belt drive systems. Chain drives are used in the transmission of mechanical energy, from driving shaft to the driven, for wider center distances, for the transmission of higher power and for a constant transmission ratio, at usually lower speeds and for the same direction of rotation [1-4]. Chain drive systems have high efficiency of transmission, requires no initial tension and can transmit power over distances larger than gear drives. Shaft center distances are relatively unrestricted. Whereas gear drive center-to-center distances are restricted to specific dimensions for a given set of gears, the center distances between two chained sprockets can vary anywhere from $50 \%$ to $300 \%$ or more of their pitch diameters [5-8].

Chain drives are relatively easy to install. Assembly tolerances are not as restrictive as those for gear drives. Chain drives are a better choice for less experienced builders working with a minimum of machine tools. Chain drives can be readily redesigned and reconfigured in comparison to gear drive systems. Chains perform better than gears under shock loading

\footnotetext{
* Corresponding author: $\underline{\text { racmil@uns.ac.rs }}$
} 
conditions [9-10]. They do not require tension on the slack side (belt drives do), thus bearing loading is reduced [11]. Chain drives spread operating loads over many teeth whereas the operating loads acting on gear drives are concentrated on one or two teeth. Careful alignment and proper lubrication are required.

\section{Various types of chain transmission}

Chain drives can be used as speed reducers $(i>1)$, as simple transmissions $(i=1)$, when the axis of rotation is just shifted, and as multipliers $(i<1)$. In the event that the chain drive needs to achieve the opposite rotation direction of the driven shaft, then another sprocket has to be positioned, but on the outside of the chain, and it represents a driven sprocket [1, $11]$.

In the case the dimension of chain gearbox should be reduced, duplex or triplex chains are used [8]. Using multi-strand transmission chain, for the same transmitted power and same axial distance, the dimensions of chain drive are slightly reduced while the angular speed is provided to be more constant, which certainly achieves some advantages.

If the center-to-center distance should be reduced, there is no need to use chain drive, so that transmission could be realised by single stage gear drive. If it is necessary to maintain the rotation direction, this can be solved by single stage gear unit with additional gear, or with double stage gear drive. However, for maintaining rotation direction, triplex chain could be used with sprockets arranged as shown in the Fig. 1. However, the efficiency of this system is not so high; usually it operates only with $1 / 3$ of the possible load carrying capacity $[5,12]$.
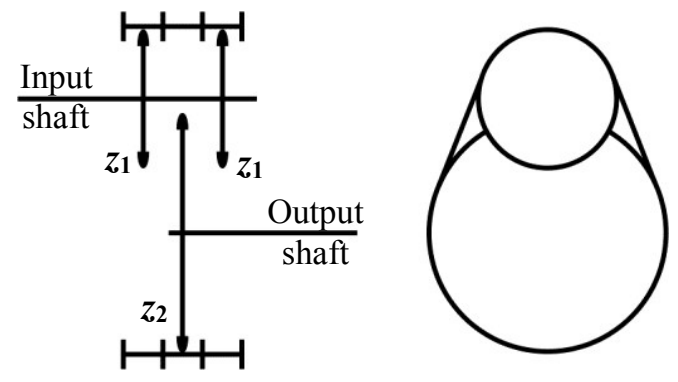

Fig.1. Characteristic solution of triplex chain transmission

Since it is a chain drive and there is no possibility of slipping, operating and kinematic transmission ratios are equal. In this case, it is also calculated in the usual way as the ratio of input and output speed, or ratio of teeth numbers of driven and driving sprocket [11]:

$$
i=\frac{n_{1}}{n_{2}}=u=\frac{z_{2}}{z_{1}}
$$

where:

$i$ - operating transmission ratio,

$n_{1}$ - input speed,

$n_{2}$ - output speed,

$u$ - kinematic transmission ratio,

$z_{1}$ - teeth number of driving sprocket,

$z_{2}$ - teeth number of driven sprocket. 
If central distance should be reduced, double stage gear drive could be used or special chain transmission with triplex chain (Fig. 2). However, the efficiency of this chain transmission is not high, usually less than $1 / 3$ of the real loadability which should be specifically examined for this type of drive $[5,12]$.
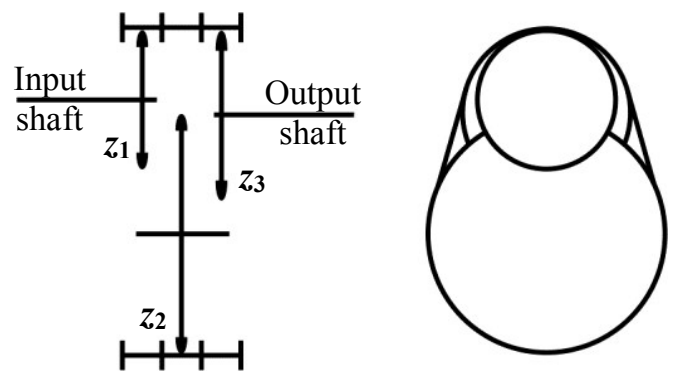

Fig.2. Characteristic solution of triplex chain transmission with small axis distance between input and output shaft

For this case, transmission ratio can be calculated in following way [11]:

$$
i=\frac{n_{1}}{n_{2}}=u=\frac{z_{2}}{z_{1}} \cdot \frac{z_{3}}{z_{2}}=\frac{z_{3}}{z_{1}}
$$

where:

$i$ - operating transmission ratio,

$n_{1}$ - input speed,

$n_{2}$ - output speed,

$u$ - kinematic transmission ratio,

$z_{1}$ - teeth number of driving sprocket,

$z_{2}$ - teeth number of inner sprocket,

$z_{3}$ - teeth number of output sprocket.

If the number of teeth of driving sprocket is less than the number of teeth of output sprocket $z_{1}<z_{3}$, the transmission reduces input speed and operates like a reducer. But, if $z_{1}>z_{3}$, the transmission increases input speed and operates like a multiplier. However, if the number of teeth of driving and output sprocket are equal $z_{1}=z_{3}$, the transmission gives the same speed as in input with the same direction, which makes no sense in transmission here $[1,9,11]$.
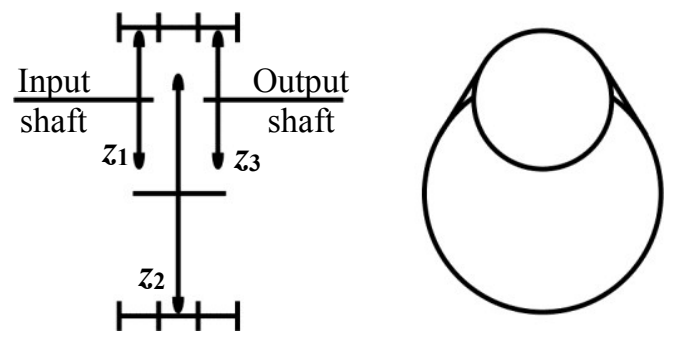

Fig.3. Characteristic solution of coaxial triplex chain transmission

In the case the transmission should be coaxial, i.e. that input and output shafts have the same axis, it could be used double stage gear drive or planetary gear drive. However, 
special chain transmission with triplex chain could be also used (Fig. 3) [8, 9]. In this case, input and output sprockets have the same diameters, but different number of teeth and thus different pitch of teeth. The difference in number of teeth should be only one tooth, which significantly affects the shape of the teeth of one of the sprocket and thus its loadability [7, $8,11]$.

Transmission ratio for this sprockets arrangement can be also calculated in the same way [11]:

$$
i=\frac{n_{1}}{n_{2}}=u=\frac{z_{2}}{z_{1}} \cdot \frac{z_{3}}{z_{2}}=\frac{z_{3}}{z_{1}}
$$

where:

$i$ - operating transmission ratio,

$n_{1}$ - input speed,

$n_{2}$ - output speed,

$u$ - kinematic transmission ratio,

$z_{1}$ - teeth number of driving sprocket,

$z_{2}$ - teeth number of inner sprocket,

$z_{3}$ - teeth number of output sprocket,

where $z_{3}=z_{1}+1$ (if the transmission should reduce the speed) or $z_{3}=z_{1}-1$ (if the transmission should increase the input speed).

Since the load carrying capacity of these gears is very small, and also transmission ratio is not high, their application is not justified [5]. However, the operating changes significantly with a slightly different chain transmission solution, i.e. using the chain drive with eccentricity (Fig. 4). The transmission ratio is increased and this solution has a little bit lower overall dimensions of the sprocket drive. For this solution, the first sprocket $z_{1}$ is stationary, i.e. firmly attached for the transmission housing. Triplex chain is enveloped around it, which activates and causes the rotation of eccentrically mounted sprocket $z_{2}$, which then causes the rotation of the sprocket $z_{3}$. The first and third sprockets have the same diameters, but different number of teeth and thus different pitch of teeth. The difference in number of teeth is only one tooth, which significantly affects the shape of the teeth of one of the sprocket and thus its load carrying capacity $[13,14,15]$.
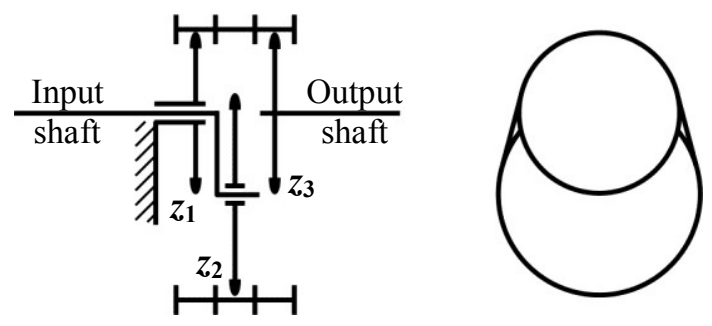

Fig.4. Characteristic solution of chain transmission with eccentricity

If the number of teeth of the first sprocket is less than the number of teeth of the third sprocket $z_{1}<z_{3}$, the chain drive reduces input speed and operates like a reducer with the same direction of rotation. Transmission ratio for this chain transmission with eccentricity can be calculated in following way $[11,13]$ :

$$
i=\frac{n_{1}}{n_{2}}=u=\frac{z_{3}}{z_{3}-z_{1}}=z_{3}
$$


where:

$i$ - operating transmission ratio,

$n_{1}$ - input speed,

$n_{2}$ - output speed,

$u$ - kinematic transmission ratio,

$z_{1}$ - teeth number of driving sprocket,

$z_{2}$ - teeth number of inner sprocket,

$z_{3}$ - teeth number of output sprocket,

where $z_{3}=z_{1}+1$.

In this case, if the number of teeth of the first sprocket is bigger than the number of teeth of the third sprocket $z_{1}>z_{3}$, the chain drive also reduces input speed and operates like a reducer, but the direction of rotation is contrary. Transmission ratio is calculated in following way $[11,13]$ :

$$
i=\frac{n_{1}}{n_{2}}=u=\frac{z_{3}}{z_{1}-z_{3}}=z_{3}
$$

where $z_{1}=z_{3}+1$.

For this type of chain drive with eccentricity, certain problem exists with the mass balance on the eccentric part, since the space for mounting counter mass is limited (Fig. 5). Existence of an imbalance at a low revolving speed is certainly not a problem; however, for a high speed revolution special attention has to be paid to the mass balance $[13,14,15]$.

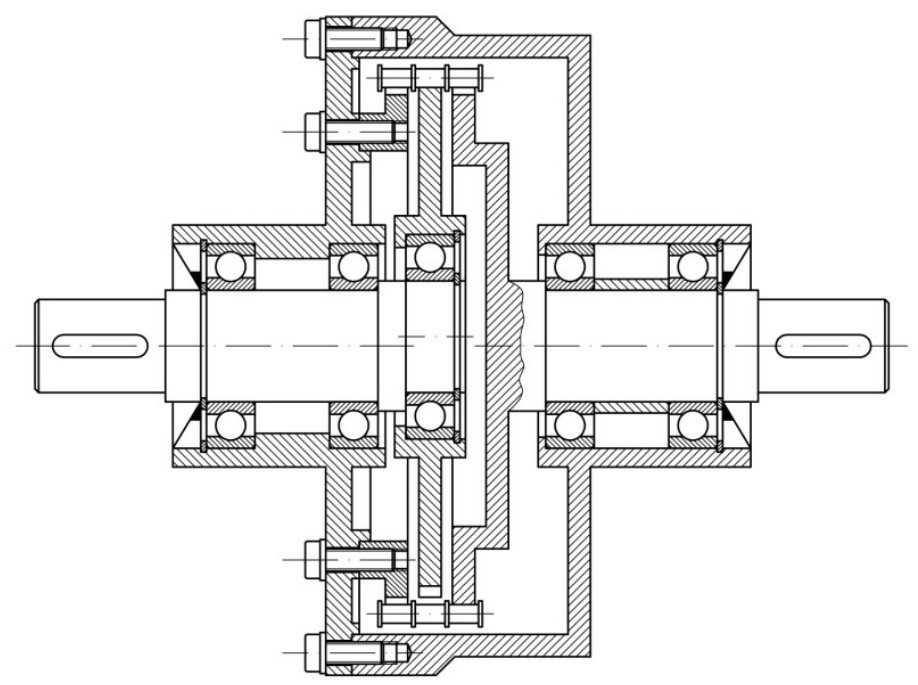

Fig.5. Assembly drawing of chain transmission with eccentricity

\section{Defining the geometry of the sprockets for chain transmission with eccentricity}

Defining the geometry of the sprocket with higher pitch can be based on the adopted chain pitch, which depends on the load of the chain drive, i.e. on the power being transmitted. Especially, it should be noted that difference in the number of teeth of the first and the third sprocket is only one. Thus, the sprocket with smaller number of teeth could have defined 
shape of teeth. The geometry of the sprockets can be easily defined by placing the circles on the same diameter corresponding to the roller diameters arranged by the same pitch as in the triplex chain (Fig. 6, 7).

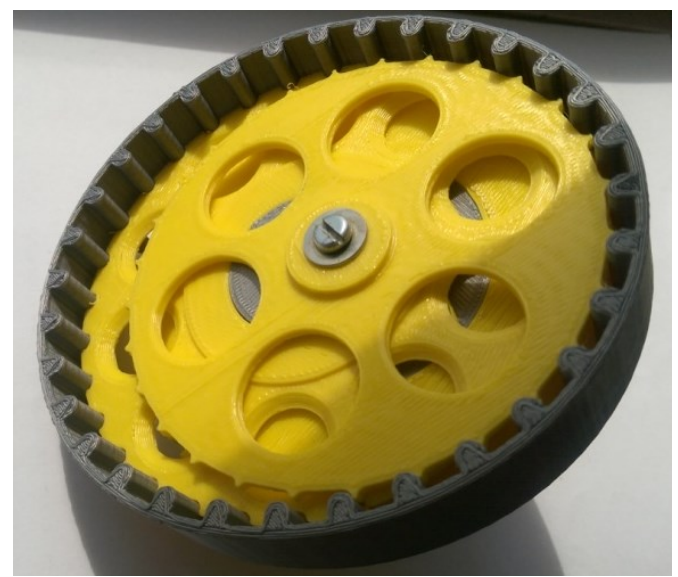

Fig.6. Chain transmission assembly with eccentricity obtained by 3D printing technology
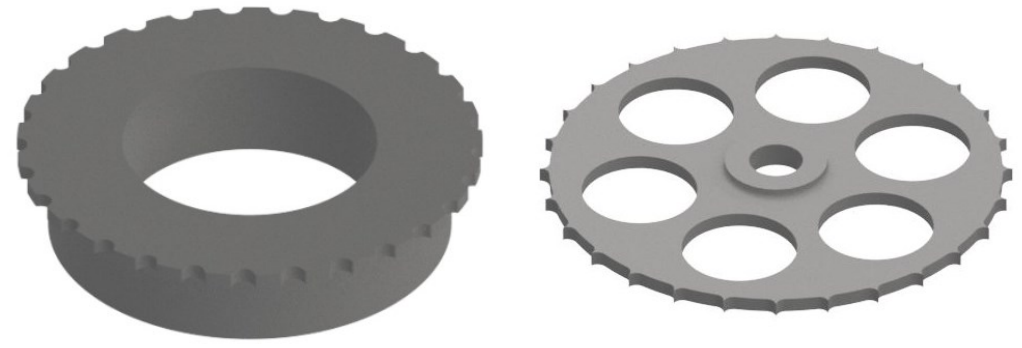

Fig.7. First and third sprocket of the chain transmission assembly with eccentricity

\section{Conclusion}

The advantages in using special chain transmission with eccentricity are reflected in the small dimensions of the chain drive compared to classical chain transmissions, simpler way of lubrication and relatively high transmission ratio. Though, its basic weaknesses are uneven revolving of the output sprocket and irrational use of chain, i.e. low carrying capacity of the chain drive since only one tooth or two teeth are in contact during operating with low carrying height. Therefore the use of this chain transmission is justified only for transmission of motion.

Timing belts can be also used for this type of transmission, but they have less load carrying capacity, so they are not considered in this analysis.

This paper is part of a research on project "Research and Development of a New Generation of Wind Generators of High Energy Efficiency" TR 35005, supported by the Ministry of Education and Science, Republic of Serbia. 


\section{References}

1. ISO 10823:2004, Guidance on the selection of chain drives

2. ISO 606:2004, Short pitch transmission precision bush chains and chain wheels

3. ISO 1275:2006, Double-Pitch Precision Roller Chains, Attachments and Associated Chain Sprockets for Transmission and Conveyors

4. S. L. Pedersen, Arche of Appl Mech 74 (2005)

5. A. Egorov, K. Kozlov, V. Belogusev, Journ of Appl Eng Sc 13, 4 (2015)

6. G.K. Ryabov, M.A. Seledzhieva, Chem and Petr Eng 49, 7-8 (2013)

7. S. Ghimisi, D. Nicula, MATEC Web of Conf 178, 06002 (2018)

8. C. Bucur, M. Marton, Proc Eng 181 (2017)

9. Y. Zhou, Exp. Tech. 40 (2016)

10. R. Protasov, S. Andrienko, A. Ustinenko, A. Bondarenko, N. Matushenko, F\&D, 1 (2017)

11. G. Niemann, Machinenelemente. Band. II (Springer Verlag, Berlin, 1975)

12. R. Velicu, R. Papuc, C.C. Gavrila, S. Popa, IOP Conf. Series: MSE 174, 012051 (2017)

13. R.N. Boggs, Design News 46, 3 (1990)

14. R.M. Peters, D. Fitzgeorge, Journ of Mech 6, 2 (1971)

15. R.M. Peters, Mech \& M Theory 7, 1 (1972) 